\title{
APPLICATIONS OF A HYPER-GRAPH GRAMMAR SYSTEM IN ADAPTIVE FINITE-ELEMENT COMPUTATIONS
}

\author{
Piotr GURGUl $^{a, *}, \mathrm{KonRAD} \mathrm{JOPEK}^{b}, \mathrm{KeShaV} \mathrm{PINGALI}^{c}$, AnNA PASZYŃSKA $^{d}$ \\ ${ }^{a}$ Dropbox Inc., 333 Brannan Street, San Francisco, CA 94107, USA \\ e-mail: piotr.gurgul@gmail.com \\ ${ }^{b}$ Department of Computer Science \\ AGH University of Science and Technology, al. Mickiewicza 30, 30-059 Kraków, Poland \\ e-mail:kjopek@gmail.com \\ ${ }^{c}$ Institute for Computational and Engineering Sciences \\ The University of Texas at Austin, Austin, TX 78712-1229, USA \\ e-mail: pingali@cs.utexas.edu \\ ${ }^{d}$ Faculty of Physics, Astronomy and Applied Computer Science \\ Jagiellonian University, ul. Łojasiewicza 11, 30-348 Kraków, Poland \\ e-mail: anna.paszynska@uj .edu.pl
}

\begin{abstract}
This paper describes application of a hyper-graph grammar system for modeling a three-dimensional adaptive finite element method. The hyper-graph grammar approach allows obtaining a linear computational cost of adaptive mesh transformations and computations performed over refined meshes. The computations are done by a hyper-graph grammar driven algorithm applicable to three-dimensional problems. For the case of typical refinements performed towards a point or an edge, the algorithm yields linear computational cost with respect to the mesh nodes for its sequential execution and logarithmic cost for its parallel execution. Such hyper-graph grammar productions are the mathematical formalism used to describe the computational algorithm implementing the finite element method. Each production indicates the smallest atomic task that can be executed concurrently. The mesh transformations and computations by using the hyper-graph grammar-based approach have been tested in the GALOIS environment. We conclude the paper with some numerical results performed on a shared-memory Linux cluster node, for the case of three-dimensional computational meshes refined towards a point, an edge and a face.
\end{abstract}

Keywords: adaptive finite element method, hyper-graph grammars, mesh-based computations.

\section{Introduction}

There have been some previous attempts to prescribe adaptive mesh-based transformations and computations by a set of graph grammars. The first work concerning this area was presented Flasiński and Schaefer (1996). Graph grammar productions were used by the authors to prescribe various transformations of regular, triangular and two-dimensional adaptive meshes. Using a quasi-context sensitive grammar turned out to have limitations in terms of adaptive meshes. As an

${ }^{*}$ Corresponding author example, application of the so-called 1-irregularity rule is contextual and cannot be modeled using context-free grammars. The 1-irregularity rule says that a finite element can be broken only once without breaking its bigger, neighboring elements. It prevents unbroken element edges from being adjacent to more than two finite elements. When an unbroken edge is adjacent to one large finite element on one side and two smaller finite elements on the other side, the approximation over these two smaller elements is constrained by the approximation of the larger element. In case the 1-irregularity rule cannot be enforced, only uniform refinements can be prescribed. 
Grabska (1993a; 1993b) introduced composite programmable graphs (CP-graphs) as a new mathematical formalism applicable to model various design processes. CP-graph grammars describe transformations of the graph representation of the domain object. Due to their contextuality, they are suitable for a wide range of problems.

The CP-graph grammar consists of a set of graph transformations, called productions. A production prescribes a replacement of a subgraph defined on its left-hand side into a new subgraph defined on its right-hand side. The $\mathrm{CP}$-graphs are very convenient for modeling the mesh transformations, since they allow for a simple definition of the embedding transformation for the replaced subgraph. This is because the transformation embedding is encoded in the production by introducing the so-called free bonds, denoting places where the replaced graph is connected through edges with the remaining parts of the graph. In the $\mathrm{CP}$-graph grammar, the same number of free bonds is assumed on both sides of the production, and the free bonds numbering is utilized to embed the replaced subgraph. CP-graph grammars were used for modeling the adaptive finite element method grids in two dimensions (Paszyński and Paszyńska 2008; Paszyński and Schaefer, 2010; Paszyński, 2009; Paszyńska et al., 2009; 2012a; 2012b), as well as in the three-dimensional case (Ryszka et al., 2015a; 2015b).

Results presented in this paper, however, were obtained leveraging the different and promising type of graph grammars called the hyper-graph grammars. Employing the hyper-graph grammar formalism instead of composite graph grammars to model mesh transformations can decrease the computational complexity of performed operations, because the number of edges and nodes in the hypergraph representing a computational mesh is much smaller than the number of edges and nodes in the corresponding composite graph. The topological structure of each quadratic element is represented here using four hypergraph nodes corresponding to four vertices and five hyperedges corresponding to its edges and interior, whereas in the CP-graph representation of a mesh element 18 graph nodes with 60 node bonds are needed. The most important factor though is the order, in which elements are browsed in the case of processing the solution computed over the mesh. CP-graphs are very good in representing hierarchies, which is exactly what is needed for a multi-frontal solver. However, accessing a neighbor element requires finding the lowest common ancestor (LCA), which is not straightforward. In the case of the solver presented in this paper, we browse elements level by level (layer by layer), which requires easy access to horizontal neighbors. This can easily be achieved by using hyper-graphs as they have a flat structure.
Hypergraphs were introduced by Habel and Kreowski (1987a; 1987b). The first application of the hypergraph grammars for modeling mesh generation was proposed by Ślusarczyk and Paszyńska (2013). In this paper we extend this application to three-dimensional generation, refinement and solution process. A hypergraph is composed of a set of nodes and a set of hyperedges to which sequences of source and target nodes can be assigned. Both nodes and hyperedges can be labeled with labels from a fixed alphabet. To represent the properties of mesh elements, the attributed hypergraphs are being used. This means that each node and hyperedge can have some attributes, such as, e.g., the polynomial order of approximation. However, since the solvers presented in this paper leverage only the $h$ version of FEM (with $p$ being fixed, yet arbitrary), labeling is not necessary and will not be used to model the solver. The hypergraphs are created from simpler hypergraphs by replacing their subhypergraphs by new hypergraphs. This operation is possible for a new hypergraph and for the subhypergraph if a sequence of so-called external nodes is specified. The hypergraph replacement is defined as follows. The subhypergraph is removed from the original hypergraph and the new hypergraph is embedded into the original hypergraph. The new hypergraph is glued to the remainder of the original hypergraph by fusing its external nodes with the corresponding external nodes in the remainder of the original hypergraph. The number of external nodes should be the same in both hypergraphs.

The modeling of the solver algorithm for two-dimensional grids was proposed by Paszyński (2016). In this paper, however, we extend the idea for adaptive three-dimensional grids.

The structure of the paper is the following. First, we introduce the hypergraph grammar for generation of three-dimensional mesh with a point singularity in Section 2.1, as well as for expressing a multi-frontal solver algorithm in Section 2.2. Next, we propose a hypergraph grammar-based linear computational cost solver for grids with point singularities in Section 3. Section 4 is devoted to theoretical analysis of the computational cost of the sequential solver. Section 5 concerns theoretical analysis of the memory usage of the sequential solver. Next, in Section 6 we present theoretical estimates of the computational complexity of the parallel shared-memory solver. Finally, Section 7 presents numerical results for sequential and parallel solvers. The paper is concluded in Section 8 .

\section{Three-dimensional grid with point singularity}

This section describes a derivation of an exemplary projection problem where we project a tangent-like function with a large gradient at one of the corners of 
the mesh. This results in a point singularity at that corner. Point singularities are very common phenomena in numerical simulations. They may result from:

- point sources on the right-hand-side of the partial differential equation (PDE) (like point heat sources in the heat transfer problem, or local point antennas in electromagnetic waves simulation problems);

- local geometrical structure of the mesh;

- a non-uniform distribution of material data (point singularities are present when three different material data meet at a given point of the domain).

In order to deliver a better solution in each iteration, we employ an $h$-refinement algorithm for this problem and run the solver in a number of iterations until the desired quality is achieved.

The $h$ refinement is a popular technique for reducing error (Bao et al., 2012; Belytschko and Tabbar, 1993). It is utilized in a large class of computational problem where the boundary layers, singularities, and/or high local gradients are encountered. In other conditions, $p$-refinement or $h p$-refinements may be preferred.

The $h$ refinement approach to improving the quality of the solution is to increase the number of elements by dividing the domain into smaller pieces. The rationale is that some sensitive regions require a lot of elements to approximate the solution fairly, whereas for others, even a relatively sparse mesh results in an acceptable error rate. The key factor in achieving satisfactory results is to find places that need fine grids. This can be done manually by predicting solution features $a$ priori (e.g., the location of the singularity) or automatically, by refining some elements dynamically and evaluating their relative error rate drop. In the latter case, the mesh is recursively subdivided until an acceptable resolution is obtained.

2.1. Hypergraph grammar model for generation and adaptation of three-dimensional mesh with point singularities. The process of generation of the three-dimensional computational mesh with hexahedral elements starts with execution of the $P_{\text {init }}$ production, presented in Fig. 1 It generates a hypergraph representing a single three-dimensional finite element. In the case of uniform mesh adaptations, we can prepare a sequence of graph grammar productions replacing the single element by a uniform cluster of elements. The model production $P_{\text {init_break }}$ presented in Fig. 2 generates a uniform mesh of eight elements. In order to get non-uniform mesh refinements, we need to enforce the afore-mentioned 1 -irregularity rule by breaking element interiors first, as is expressed by production $P_{\text {break_int }}$ presented in Fig. 3. An example execution of the production over the eight finite element mesh is presented in Fig. 4. In the case of faces,

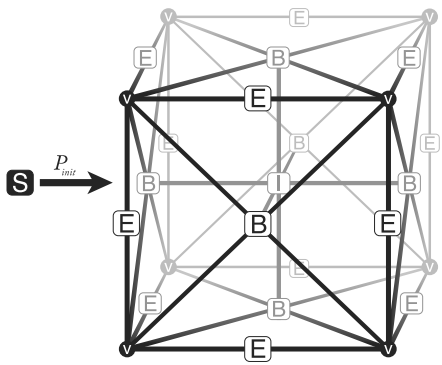

Fig. 1. Initial production $P_{\text {init }}$ that generates a single cubic element.
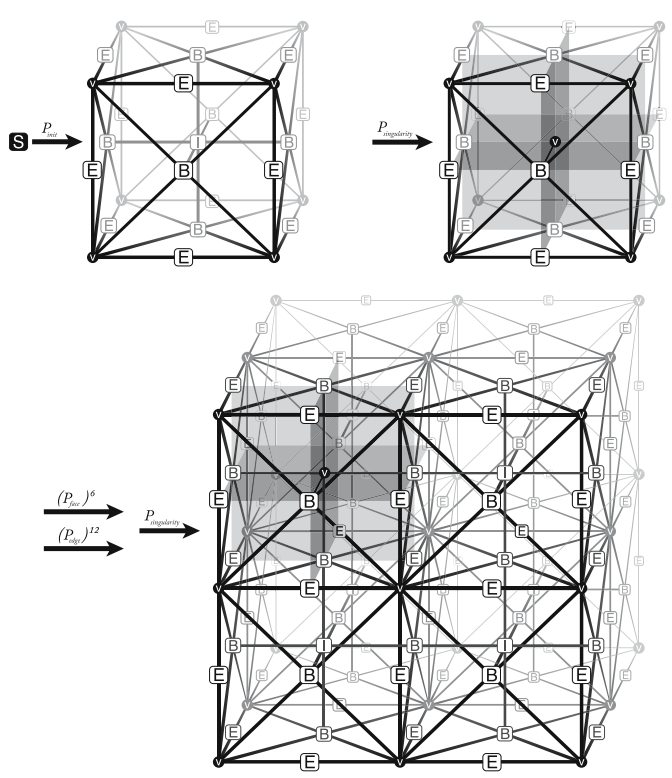

Fig. 2. Production breaking a single element into eight elements.

a face can be broken only if two adjacent interiors have already been broken, or one adjacent interior has been broken and the face is located on the boundary of the mesh. The case is illustrated with production $P_{\text {break_face }}$ presented in Fig. 5 Finally, edges can be broken only if all adjacent faces have already been broken, or the edge is located on the boundary (and in such a scenario we need to check fewer adjacent faces). This is illustrated in Fig.6 by production $P_{\text {break_edge }}$.

\subsection{Multi-frontal solver algorithm prescribed by hy-} pergraph grammars. In order to apply the concept to a frontal solver algorithms, a simple 2D two finite element example will be used. The domain $\Omega$ is described by two elements and fifteen nodes-two interiors, seven edges and six vertices (cf. Fig. 7). In the 2D FEM we utilize basis functions related to element nodes. In this example, as presented in Fig. 8 we have linear basis functions 

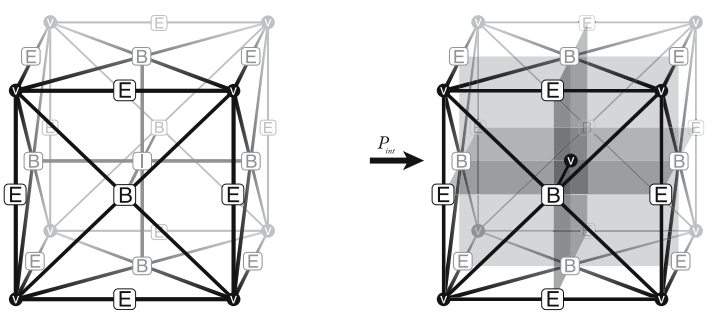

Fig. 3. Production breaking an interior of a single element.

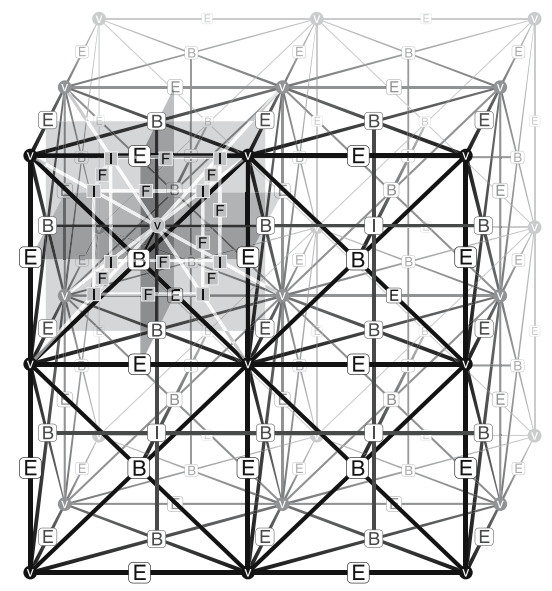

Fig. 4. Eight-element mesh after breaking the interior of the front element.

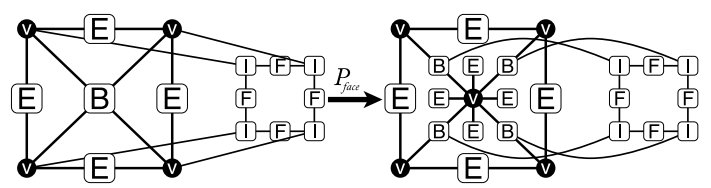

Fig. 5. Production $P_{\text {face }}$ that breaks a face.

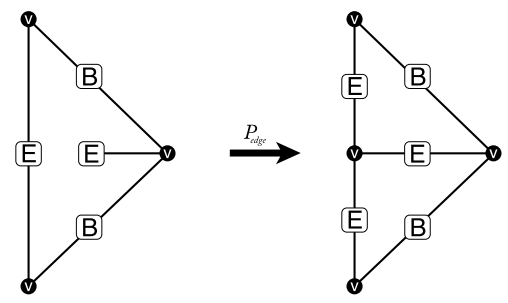

Fig. 6. Production $P_{\text {edge }}$ that breaks an edge.

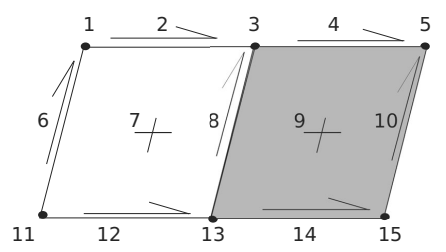

Fig. 7. Sample computational domain for the frontal solver. related to element vertices, i.e., to nodes $1,3,5,11$, 13 and 15 , quadratic basis functions related to element edges, namely to nodes 2, 4, 6, 8, 10, 12 and 14, as well as quadratic basis functions related to element interiors, namely to nodes 7 and 9 . We construct the matrix by integrating multiplications of these basis functions or their derivatives over a domain. Thus, matrix rows and columns correspond to basis functions and matrix entries correspond to multiplications of pairs of basis functions. Interior basis functions have support over a given element only, edge basis functions have support spread over one or two elements, vertex basis functions also have support spread over one or many elements.

The frontal solver introduced by Irons (1970) browses finite elements in a user-determined, arbitrary order. Due to its nature, it is sequential. Nodes (degrees of freedom) are aggregated into so-called frontal matrices.

Instead of generating just one large FEM matrix, it generates small matrices, called element frontal matrices. These matrices are obtained by integrating basis functions
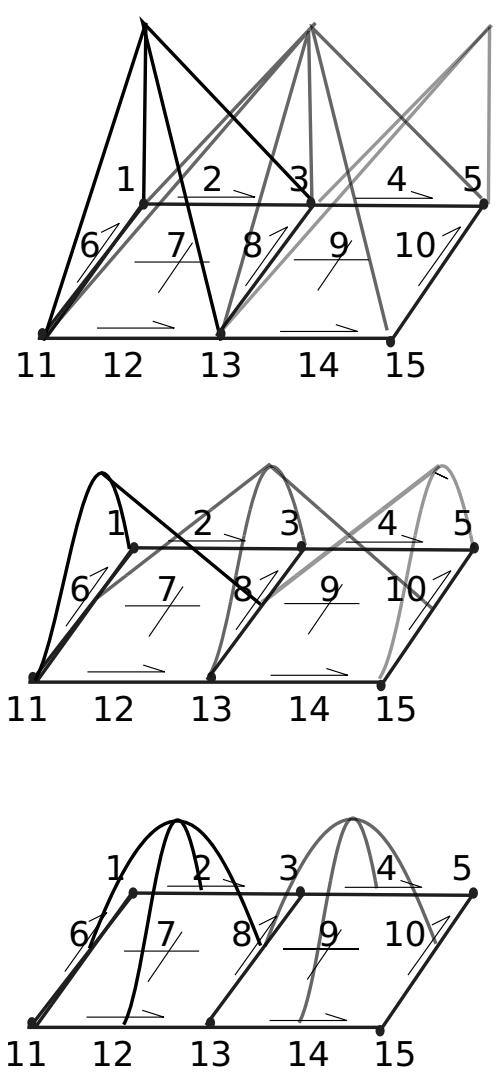

Fig. 8. Example basis functions spread over element nodes: the basis function associated with vertex node 1 (black), vertex node 3 (dark gray) and vertex node 5 (light gray) (a), the basis function associated with edge node 6 (black), edge node 8 (dark gray) and edge node 10 (light gray) (b), the basis function associated with interior node 7 (black) and interior node 9 (dark gray) (c) . 


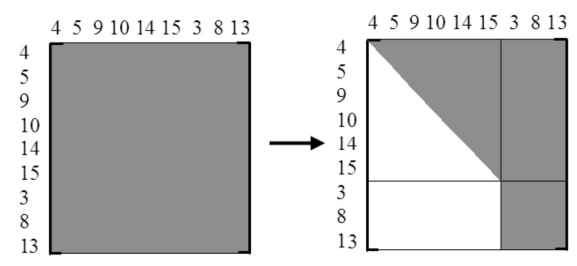

Fig. 9. Processing of the right element by the frontal solver.

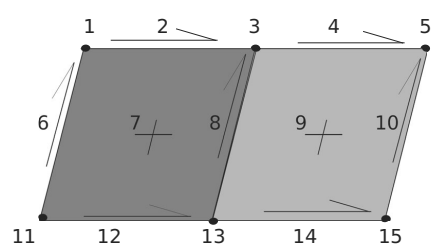

Fig. 10. Processing of the left element by the frontal solver.

over a given element. Thus, some entries in the element frontal matrix are fully assembled, and some are not. The row of the frontal matrix is called fully assembled, if all of its entries (integrals of products of pairs of basis functions) have been fully computed. This happens if both basis functions have support over a given element only, or the first of the basis functions has support over a given element only (since even the second basis functions also has support over some other element, its product with the first basis function is also zero). The fully assembled rows are self-contained and can be eliminated by the solver algorithm at any time. If a basis function has support defined only over a single element, we say that the node is reduced to the element only, and it has all its contributions already present in the element frontal matrix.

The aim of the frontal solver is to keep the frontal matrix as small as possible. To this end, it analyses the connectivity of the nodes and performs partial forward elimination of the fully assembled nodes. Fully assembled nodes have all of their contributions already present in the matrix, so no additional knowledge is necessary to eliminate corresponding rows. Its mechanisms are presented using an example of a two-element mesh. Since the order is arbitrary, we decided to add the nodes of the right element to the matrix first. Nodes 4, 5, 9, 10, 14 and 15 are proprietary to the right element and thus, we can call them fully assembled. Nodes 3, 8 and 13 are shared with the left element, and hence, will not be fully assembled until the frontal matrix associated with the left element is not merged with the frontal matrix associated with the right element.

The frontal solver browses elements one by one. It starts with generating a frontal matrix for the right element, for all the nodes $(4,5,9,10,14,15,3,8,13)$. We put the fully assembled nodes $(4,5,9,10,14,15)$ in the upper part of the matrix. We perform partial forward elimination, eliminating all fully assembled nodes (4, 5,
9, 10, 14, 15). This is shown in Fig. 9 The upper triangular part of the frontal matrix has to be stored for future backward substitution. What is left is the reduced frontal matrix associated with nodes $(3,8,13)$, which are not yet fully assembled. Such a reduced matrix is called the Schur complement matrix. The solver now moves to the left element (Fig. 10) and generates its frontal matrix $(3,8,13,1,2,6,7,11,12)$, which is followed by adding the contribution that remained after processing the right element. This is illustrated in Fig. 11. Now, all nodes are fully assembled in a single matrix, so it is possible to perform full forward elimination.

The process is followed by backward substitution, browsing elements in reverse order. The solver takes advantage of the upper triangular form of the frontal matrix and computes the solution at each node, one by one. Such an approach allows us to keep the size of the matrix as small as possible by eliminating unknowns as soon as possible. Unfortunately, as mentioned before, in this case only the matrix operations can be parallelized due to the nature of the algorithm.

The multi-frontal solver introduced by Duff and Reid $(1983$; 1984) is the state-of-the-art direct solver algorithm for solving systems of linear equations, which is a generalization of the frontal solver algorithm (Irons, 1970). However, in the case of a multi-frontal solver, connectivity analysis is performed using a so-called elimination tree. A computational domain is decomposed into hierarchical subdomains, which account for the elimination tree (Fig. 12). The construction of the elimination tree for an arbitrary mesh is a complex task per se. It is done by constructing the graph representing the connectivities in the mesh, which is followed by

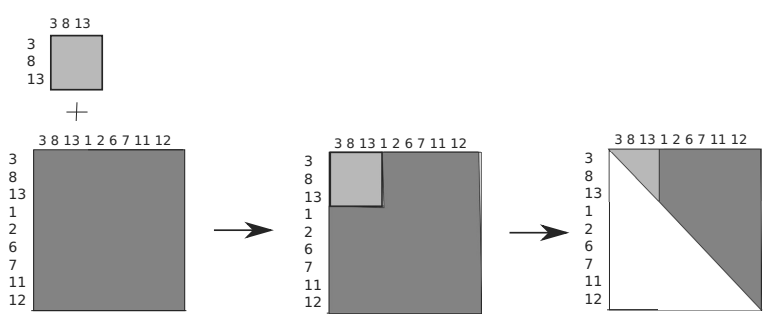

Fig. 11. Upper triangular form of the frontal matrix after processing the second element.
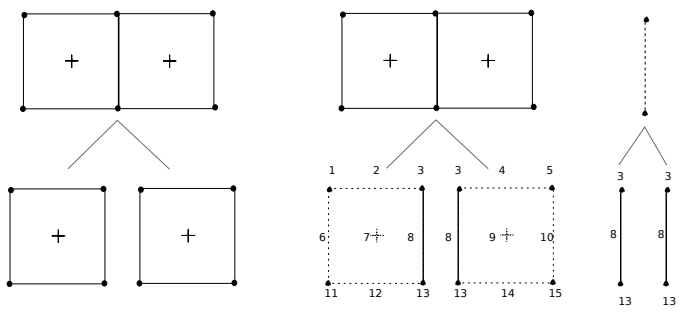

Fig. 12. Domain decomposed into an elimination tree. 
running graph partitioning algorithms such as nested dissections from METIS library (Karypis and Kumar, 2009).

Usually, commercial solvers like the MUMPS (Duff and Reid, 1983; 1984) solver are not aware of the structure of the mesh, and they need to reconstruct the connectivity pattern by analysing the sparsity pattern of the matrix submitted to the solver. Note that such a matrix is already in its the global form, after the assembly of all element frontal matrices. Another method for construction of elimination trees is presented by Aboueisha et al. (2017).

In the multi-frontal approach, the solver generates a frontal matrix for each element of the mesh. This is illustrated in Figs. 13 and 14. It eliminates fully assembled nodes within each frontal matrix, and merges the resulting Schur complement matrices at the parent level of the tree. This is illustrated in Fig. 15. The key difference with respect to the frontal matrix is that at the parent level the solver works with a smaller matrix, which is a $3 \times 3$ matrix obtained from the two Schur complements computed at its son nodes. In other words, the frontal matrix assembles element frontal matrices to a single frontal matrix and eliminates what is possible from the single matrix, while the multi-frontal solver utilizes multiple frontal matrices and thus allows us to reduce the size of the matrices at the parent nodes of the tree.

The first graph grammar productions are responsible for generation of the frontal element matrices. This is done by productions $P_{\text {agreg_int }}$ responsible for generation of the matrix entries associated with interior nodes, $P_{\text {agreg_boundary }}$ and $P_{\text {agreg_face }}$ responsible for generation of matrix entries associated with boundary and interior faces, $P_{\text {agreg_edge }}$ and $P_{\text {agreg_vertex }}$ responsible for generation of matrix entries associated with element edges and vertices. These graph grammar productions are illustrated in Fig. 16

Having assembled the frontal matrix, it is now
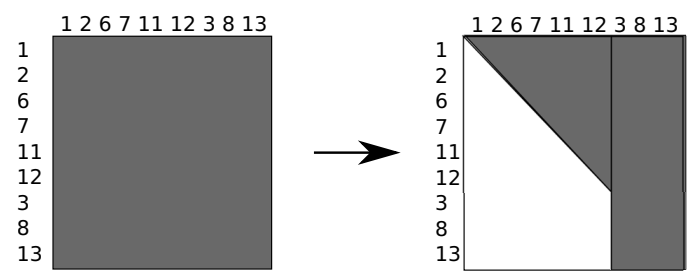

Fig. 13. Partial forward elimination on the left element.

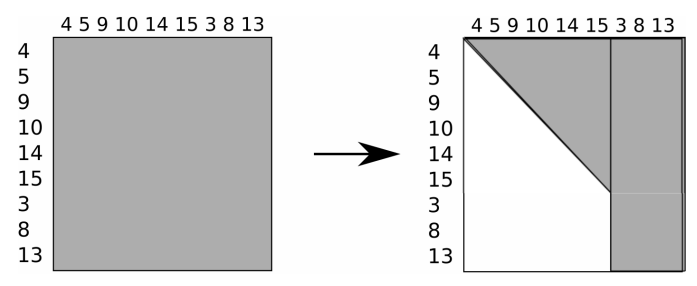

Fig. 14. Partial forward elimination on the right element.

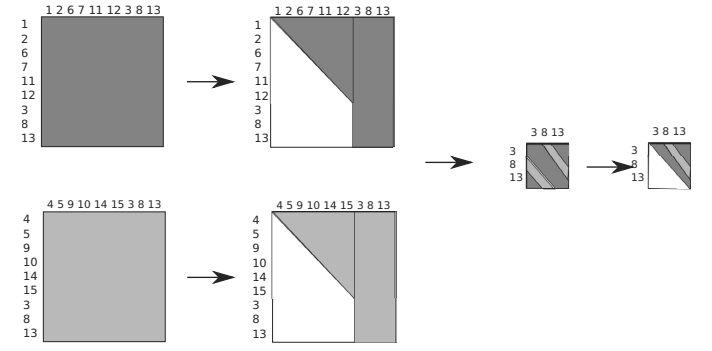

Fig. 15. Full forward elimination of the interface problem matrix.

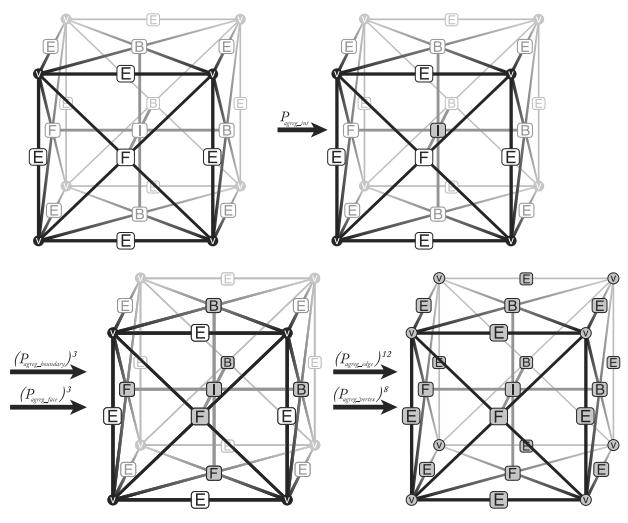

Fig. 16. Productions for assembly of an element frontal matrix.

possible to start elimination of the fully assembled nodes. We can eliminate the interior node, which is denoted by production $P_{\text {elim_int }}$ presented in Fig. 17 we can also eliminate boundary faces, which is denoted by production $P_{\text {elim_face }}$ as well as boundary edges and vertices, compare productions $P_{\text {elim_edge }}$ and $P_{\text {elim_vertex }}$ in Fig. 17

Having adjacent elements with frontal matrices and eliminated interior and boundary nodes, we can now merge the frontal matrices into one matrix and eliminate fully assembled nodes from the common face. It is expressed by productions $P_{\text {merge_eliminate }}$ illustrated in Fig. 18. This procedure of merging frontal matrices and eliminating fully assembled nodes located on common faces is repeated until all the nodes in the mesh are eliminated.

\section{Linear computational cost solver for three-dimensional meshes with point singularities}

In the case of a mesh with point singularities, as the one presented in Fig. 19, we can take advantage of the multi-level structure of the computational grid in the following way: We start with the grid presented in the right panel in Fig. 2 The first step is to execute the multi-frontal solver algorithm for all elements, except the elements located closest to the point singularity.

In order to create element frontal matrices for all 

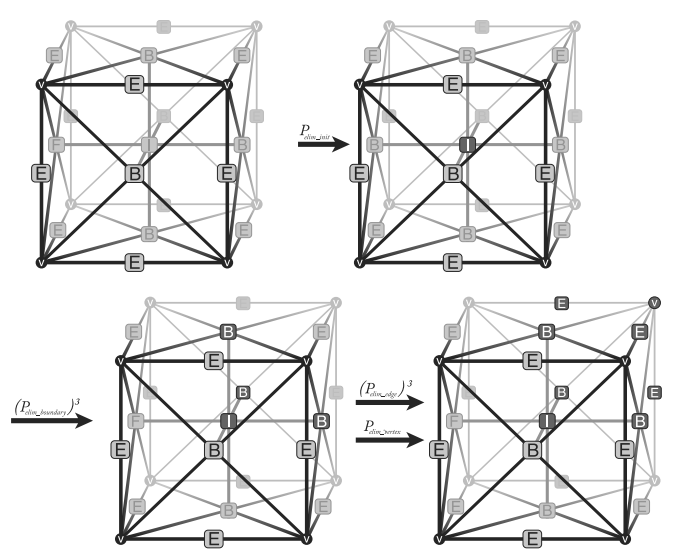

Fig. 17. Squashed productions $P_{\text {elim_int }}, P_{\text {elim_boundary }}, P_{\text {elim_edge }}$ and $P_{\text {elim_vertex }}$ that execute elimination of the interior, boundary nodes, edges and an internal vertex.
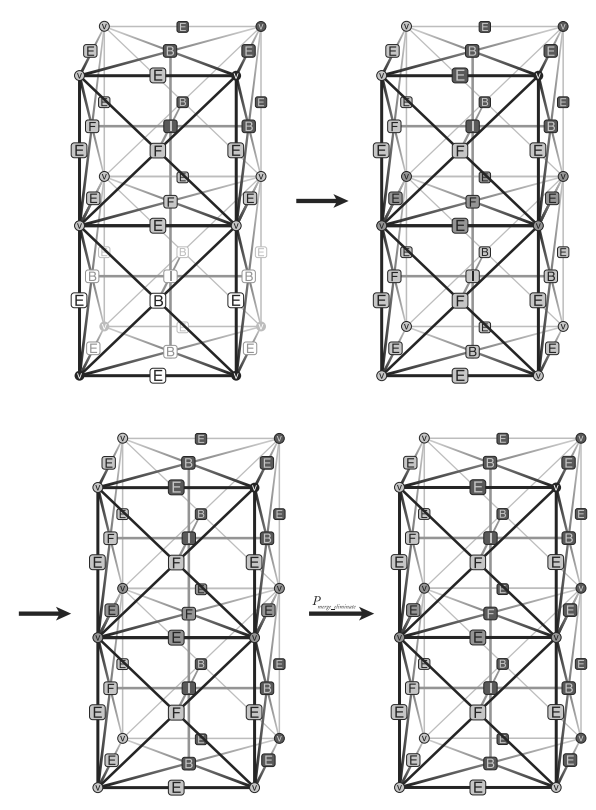

Fig. 18. Production $P_{\text {merge_eliminate }}$ for merging two frontal matrices and elimination of fully assembled nodes from a common face.

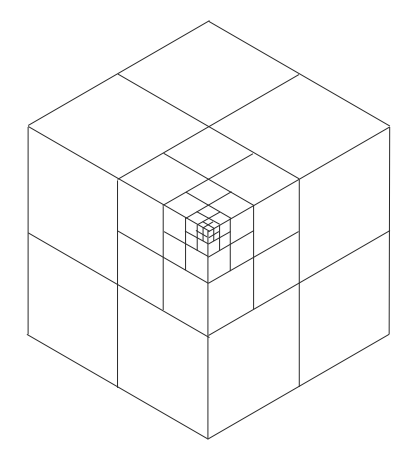

Fig. 19. Three-dimensional mesh with a single point singularity. elements on the top level we execute the following chain of productions: $P_{\text {agreg_init }} \mapsto P_{\text {agreg_boundary }} \mapsto$ $P_{\text {agreg_face }} \mapsto \quad P_{\text {agreg_edge }} \mapsto P_{\text {agreg_vertex. }}$ The next step is to eliminate entries that resulted from the previously aggregated element contributions, which can be achieved by executing the following chain of productions: $P_{\text {elim_boundary }} \mapsto \quad P_{\text {elim_edge }} \mapsto$ $P_{\text {elim_vertex }}$.

Finally, we merge the frontal matrices, by executing productions $P_{\text {merge_eliminate }}$ as many times as necessary to end up with the interface of the top level with respect to the next level, as presented in Fig. 20, We store the Schur complement matrix associated with the interface.

At this point, we can aggregate the frontal matrix associated with the element nearest to the point singularity, by executing productions $P_{\text {agreg_init }} \mapsto$ $P_{\text {agreg_boundary }} \mapsto P_{\text {agreg_face }} \mapsto P_{\text {agreg_edge }} \mapsto$ $P$ agreg_vertex .

As the next step, we can merge the element frontal matrix with the Schur complement matrix, by executing production $P_{\text {merge_with_Schur. }}$ This results in a fully assembled matrix and we can solve the problem close to the singularity.

If a more accurate solution is desirable, we can break the element neighboring the singularity by executing the production $P_{\text {break singularity }}$ presented in Fig. 21, preserving the Schur complement adjacent to the broken element.

At this point, we continue solving the problem over the newly refined elements by executing the following chain of productions: $P_{\text {agreg_init }} \mapsto P_{\text {agreg_boundary }} \mapsto$ $P_{\text {agreg_face }} \mapsto P_{\text {agreg_edge }} \mapsto P_{\text {agreg_vertex }}$ that generate the element frontal matrices, followed by productions $P_{\text {elim_boundary }} \mapsto \quad P_{\text {elim_edge }} \mapsto P_{\text {elim vertex }}$, together with $P_{\text {merge_with_Schur }}$ reutilizing the Schur complement matrix for the interface with the other part of the mesh.

\section{Computational complexity of a sequential hypergraph grammar-based solver for three-dimensional meshes with point singularities}

As a diligent estimation of the exact computational cost for the three-dimensional version of the solver would be a very strenuous task, we limit ourselves to a rough approximation of the computational complexity, ignoring the constants. Note that $p$ is assumed to be constant over the entire domain. In fact, due to numerical unstability, $p$ very rarely exceeds 10 and hence this assumption is fair. In case of the $h p$ version of the FEM algorithm, where both $h$ and $p$ are variable, we can assume that $p=p_{\max }=$ const to approximate the upper boundary of the cost.

Lemma 1. The computational complexity of the sequential solver with respect to the number of the degrees of 


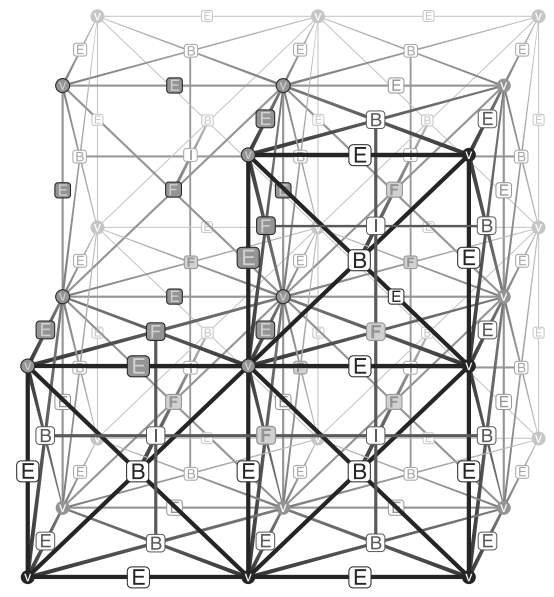

Fig. 20. Interface between layers.
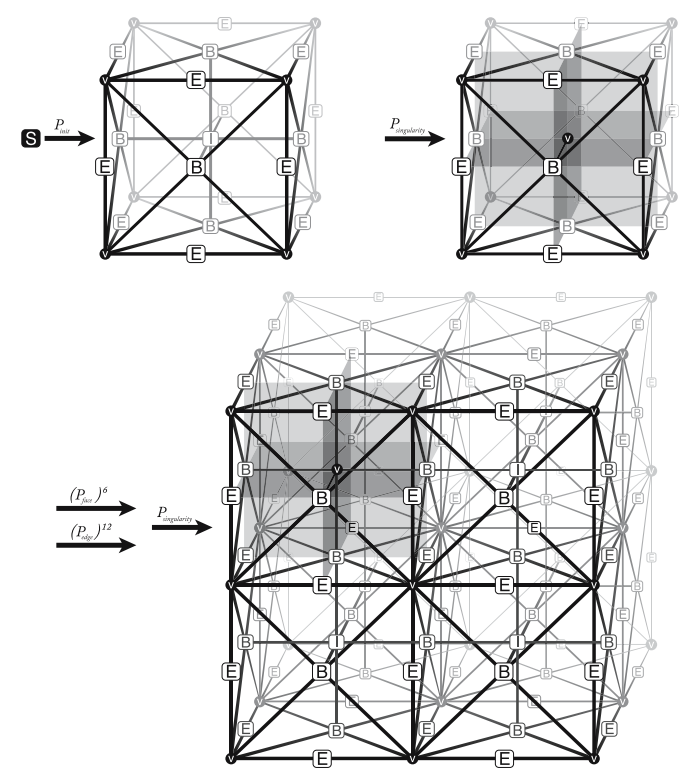

Fig. 21. Hypergraph grammar derivation of a grid with a point singularity.

freedom $N$ and the polynomial order of approximation $p$ for the three-dimensional grid with a point singularity is equal to $T(p, N)=O\left(N p^{6}\right)$.

Proof. A three-dimensional element has $O(p)$ degrees of freedom over an element edge, $O\left(p^{2}\right)$ degrees of freedom over an element face and $O\left(p^{3}\right)$ degrees of freedom over an element interior. The number of base functions spanned over the element vertices is constant and hence independent of $p$. The computational complexity of the elimination of the interior-related degrees of freedom is of the order of $O\left(\left(p+p^{2}+p^{3}\right)^{2} p^{3}\right)=O\left(p^{9}\right)$. The computational complexity of the static condensation is of the order of $O\left(N_{e} p^{9}\right)$, where $N_{e}$ denotes the number of elements.

The remaining degrees of freedom over the faces and edges are eliminated level by level (layer by layer), and the computational complexity of elimination of a single level is of the order of $O\left(\left(p^{2}+p\right)^{3}\right)=O\left(p^{6}\right)$. The number of elements $N_{e}$ is of the order of $O\left(N_{e}\right)=O\left(N / p^{3}\right)$, and the number of levels $k$ is of the order of $O(k)=$ $O\left(N / p^{3}\right)$. Thus the total computational complexity is of the order of $O\left(N_{e} p^{9}+k p^{6}\right)=O\left(N p^{6}+N p^{3}\right)=$ $O\left(N p^{6}\right)$, which completes the proof.

The proven complexity depends on $p$ that in theory is variable. However, as mentioned above, in practice it very rarely exceeds 10 due to severe numerical problems caused by high order polynomials. For the case of $h p$-refinements, where the polynomial orders of approximations vary, we can use the above estimate as the upper bound for the computational cost, assuming the uniform distribution of the maximum utilized polynomial order of approximation.

\section{Memory usage of a hypergraph grammar-based solver for three-dimensional meshes with point singularities}

Memory usage in case of the hypergraph grammar driven solver for three-dimensional meshes with point singularities remains linear with respect to the number of the degrees of freedom $N$. The order of memory usage is roughly estimated in the lemma below.

Lemma 2. Memory usage $M$ of the solver with respect to the number of degrees of freedom $N$ and polynomial order of approximation $p$ for the three-dimensional grid with a point singularity is of the order of $M(N, p)=O\left(N p^{3}\right)$.

Proof. A three-dimensional element contains $O(p)$ degrees of freedom over element edges, $O\left(p^{2}\right)$ degrees of freedom over element faces and $O\left(p^{3}\right)$ degrees of freedom over element interiors. The memory usage complexity of storing an element frontal matrix is of the order of $O\left(\left(p+p^{2}+p^{3}\right)^{2}\right)=O\left(p^{6}\right)$. The total memory usage complexity of storing element frontal matrices is of the order of $O\left(N_{e} p^{6}\right)$. The matrices at higher levels contain contributions from faces and edges and the memory usage complexity of storing such a matrix is of the order of $O\left(\left(p^{2}+p\right)^{2}\right)=O\left(p^{4}\right)$. The number of elements $N_{e}$ is of the order of $O\left(N_{e}\right)=O\left(N / p^{3}\right)$, and the number of levels $k$ is of the order of $O(k)=O\left(N / p^{3}\right)$. Thus the total memory usage complexity is of the order of $O\left(N_{e} p^{6}+k p^{3}\right)=O\left(N p^{3}+N\right)=O\left(N p^{3}\right)$. 


\section{Theoretical estimates of computational complexity for parallel shared memory machine solver execution}

In this section we roughly estimate a theoretical computational complexity of the fastest version of the solvers presented in this paper which is the parallel hypergraph grammar solver for three-dimensional meshes with point singularities.

We assume that execution takes place on a shared memory machine. For a message passing parallelism model, the cost of communication needs to be included. Another important assumption is the infinite number of cores, so that the scalability is unrestricted. While it is an idealized scenario, in reality it would be enough to find a right balance between the problem size and the amount of cores available to optimize the performance. In the worst case of severe misconfiguration, the complexity of this algorithm will be reduced to linear. A good analogy would be a comparison to a binary search tree which on the average offers logarithmic time for all basic operations, but in its extreme, imbalanced version, the complexity deteriorates to linear.

Lemma 3. The computational complexity of the parallel solver with respect to the number of degrees of freedom $N$ and polynomial order of approximation $p$ for the threedimensional grid with a point singularity is of the order of $O\left(p^{6} \log \left(N / p^{3}\right)\right)$.

Proof. A three-dimensional element (see Fig. 1) contains $O(p)$ degrees of freedom over element edges, $O\left(p^{2}\right)$ degrees of freedom over element faces and $O\left(p^{3}\right)$ degrees of freedom over element interiors. The number of the degrees of freedom over vertices is obviously constant and it does not depend on $p$.

The computational complexity of elimination of interior degrees of freedom is of the order of $O\left(p^{3}(p+\right.$ $\left.\left.p^{2}+p^{3}\right)^{2}\right)=O\left(p^{9}\right)$, since we eliminate $p^{3}$ interior nodes from the element matrix with all element degrees of freedom of order of $p+p^{2}+p^{3}$ (subtract $p^{3}$ rows from an element matrix). The degrees of freedom over the remaining faces and edges are eliminated level by level (layer by layer), and the computational complexity of their elimination over a single level is of the order of $O\left(\left(p^{2}+p\right)^{3}\right)=O\left(p^{6}\right)$. This is because we have the order of $p^{2}+p$ degrees of freedom on the interface and the same order of degrees of freedom in the entire layer. In parallel, we construct an elimination tree with $O(\log (k))$ levels, where $k$ is the previously defined number of the refinement levels. In particular, the number of levels $k$ is of the order of $O(k)=O\left(N / p^{3}\right)$ and thus $O(\log (k))=O\left(\log \left(N / p^{3}\right)\right)$. This is why the computational complexity of the parallel solver is $O\left(p^{9}+\right.$ $\left.p^{6} \log \left(N / p^{3}\right)\right)=O\left(p^{6} \log \left(N / p^{3}\right)\right)$. This is because we perform parallel elimination of elements interior, followed by the elimination of the refinement levels on particular layers of the tree with depth $O\left(\log \left(N / p^{3}\right)\right)$. This completes the proof.

\section{Numerical results for a multi-thread shared memory GALOIS solver}

To conclude the considerations above, we present a series of numerical results illustrating the performance of the described solver. We used the GALOIS environment (Goik et al., 2014; Paszyńska et al., 2015; Pingali et al., 2011) for solver implementation. We first illustrate the linear scalability of the sequential solver when executed on the sequence of grids refined towards point 19 , edge 22. and face 23 The scalability of the sequential code is illustrated in Figs. 2427 for the point singularity, in Figs. 28, 31 for the edge singularity, and in Figs. 32,35 for the face singularity.

The mesh with a point singularity yields linear computational cost, which can be read from Figs. 24 27. for the grids with uniform polynomial order of approximations $p=2,3,4,5$. The linear computational cost of the sequential solver is also obtained for the mesh with an edge singularity, which can be read from Figs. 28, 31 for the polynomial orders of approximations $p=2,3,4,5$. Finally, the mesh with face singularity

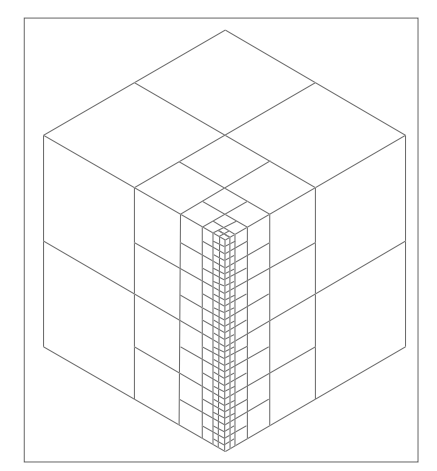

Fig. 22. Mesh refined towards an edge.

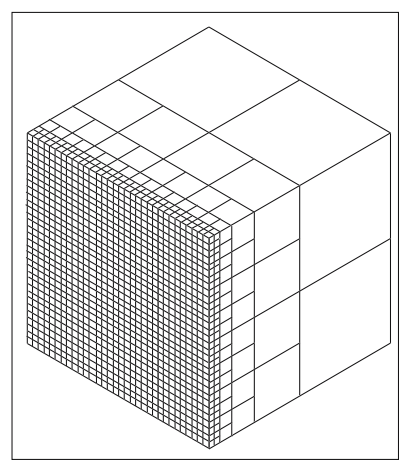

Fig. 23. Mesh refined towards a face. 
also delivers a linear cost, for different polynomial orders, which can be read from Figs. 32, 35. For very small grids, and for quadratic basis functions, the computational problem is very small, and there are some oscillations in the time measurements.

From these plots we can observe, that if the problem size becomes large enough, we recover linear computational cost not only for the point singularity, but also for the edge and the face.

Second, we illustrate the scalability of the parallel solver when executed on the fixed mesh refined towards points 36, 39, edges 40, 43, and faces 44,47, The experiments were performed on a GILBERT shared-memory Linux cluster node using up to 16 cores.

We can observe the logarithmic decrease in the computation time for the grid with a point singularity, when increasing the number of cores, as presented in Figs. 36 39, for different polynomial orders of approximation. A similar logarithmic decrease can be observed for the grids with edge singularities, as presented in Figs. 40, 43, and for the grids with face singularities, as presented in Figs. 4447

We would like to point out that horizontal lines in Figs. 24 35 presenting the sequential results contain the problem size, while the horizontal lines in Figs. 364 47 presenting the parallel results contain the number of utilized cores. Each parallel experiment has been executed on a single mesh with fixed size. The size of the mesh can be obtained by looking at the sequential computations, where the sequential execution time matches the parallel execution time with a single core. Notice that the theoretical result concerning the logarithmic computational cost of the parallel algorithm assumes an infinite number of cores, and we are restricted here to the case of 16 cores only, so producing an ideal logarithmic estimate is rather impossible in practice. From these plots we can observe that if the problem size becomes large enough (if we increase the polynomial order over the mesh nodes), the parallel code scales very well. For low computational cost grids (low polynomial order over the mesh nodes) the tasks are not heavy and the scheduling is more expensive than execution.

\section{Conclusions}

The main and most significant achievement of this work is creation of models applicable to a wide class of adaptive algorithms. The formal methodology used to achieve this goal was the hypergraph grammar formalism. All the graph grammar productions presented in this work can also be applied to three-dimensional grids with arbitrary refinements. However, for the sake of simplicity, we restricted ourselves here to the subset of hypergraph grammar productions expressing the grids with single point singularities. Nevertheless, in the numerical sections

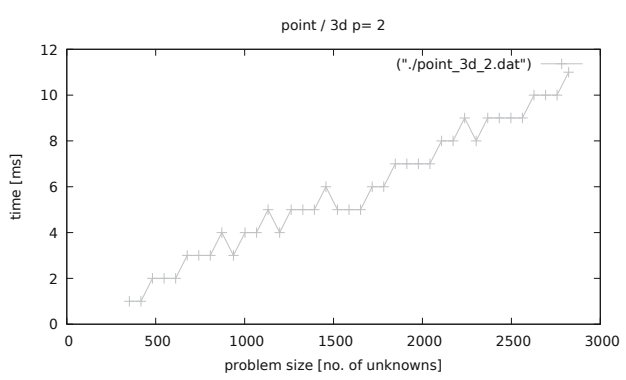

Fig. 24. Scalability of sequential hypergraph grammar-based execution over the sequence of meshes refined toward the point singularity, with uniform $p=2$.

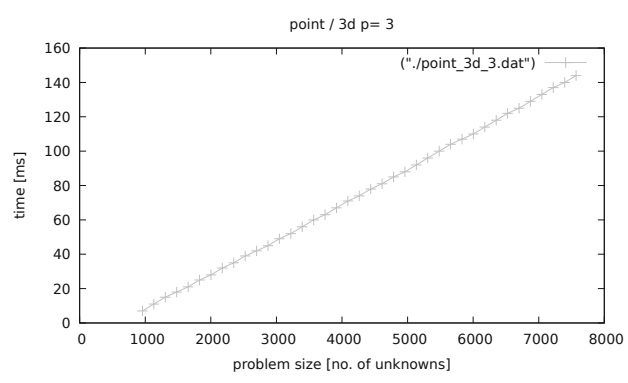

Fig. 25. Scalability of sequential hypergraph grammar-based execution over the sequence of meshes refined toward the point singularity, with uniform $p=3$.

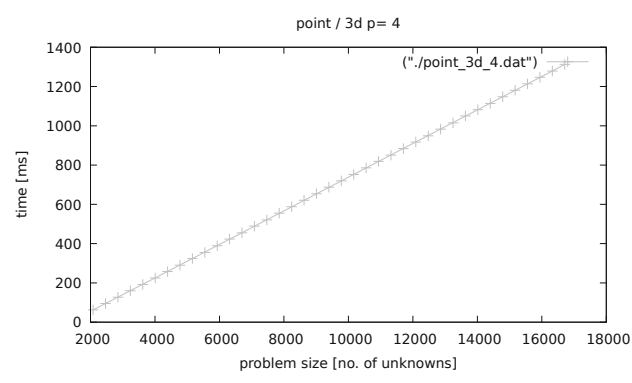

Fig. 26. Scalability of sequential hypergraph grammar-based execution over the sequence of meshes refined toward the point singularity, with uniform $p=4$.

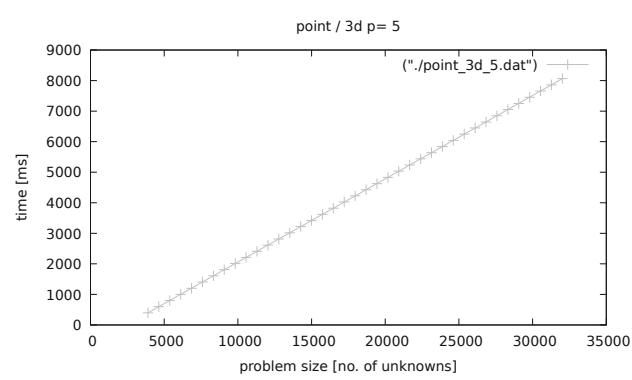

Fig. 27. Scalability of sequential hypergraph grammar-based execution over the sequence of meshes refined toward the point singularity, with uniform $p=5$. 


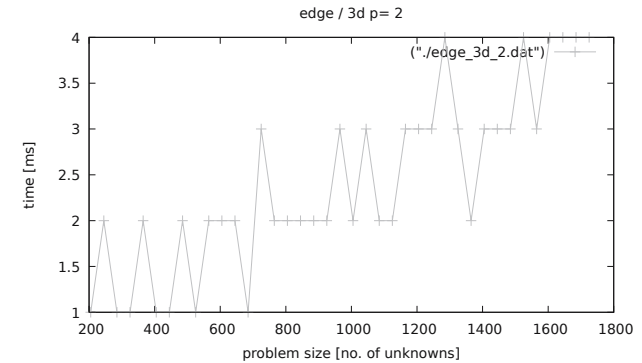

Fig. 28. Scalability of sequential hypergraph grammar-based execution over the sequence of meshes refined toward the edge singularity, with uniform $p=2$.

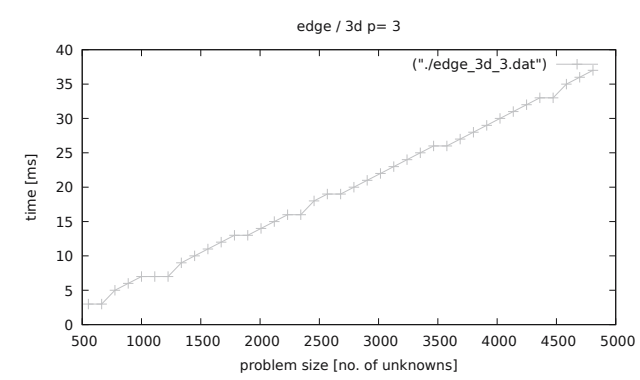

Fig. 29. Scalability of sequential hypergraph grammar-based execution over the sequence of meshes refined toward the edge singularity, with uniform $p=3$.

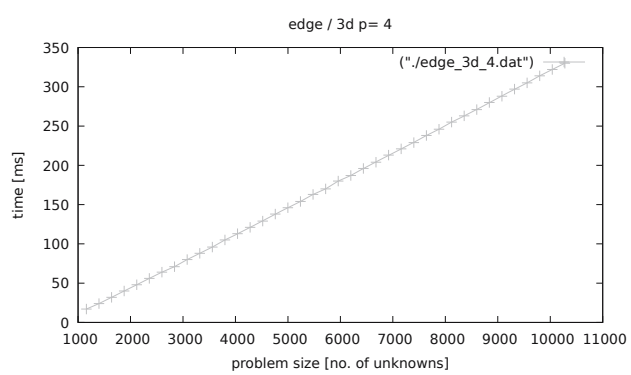

Fig. 30. Scalability of sequential hypergraph grammar-based execution over the sequence of meshes refined toward the edge singularity, with uniform $p=4$.

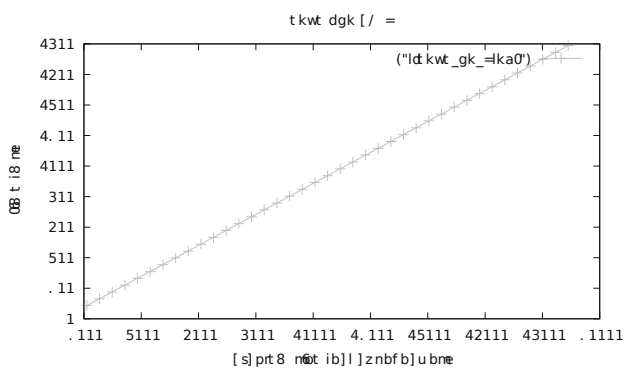

Fig. 31. Scalability of sequential hypergraph grammar-based execution over the sequence of meshes refined toward the edge singularity, with uniform $p=5$.

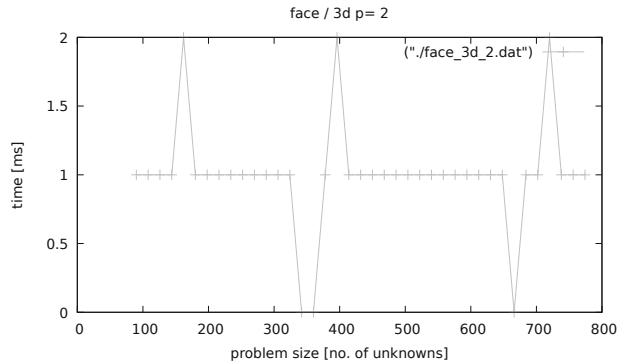

Fig. 32. Scalability of sequential hypergraph grammar-based execution over the sequence of meshes refined toward the face singularity, with uniform $p=2$.

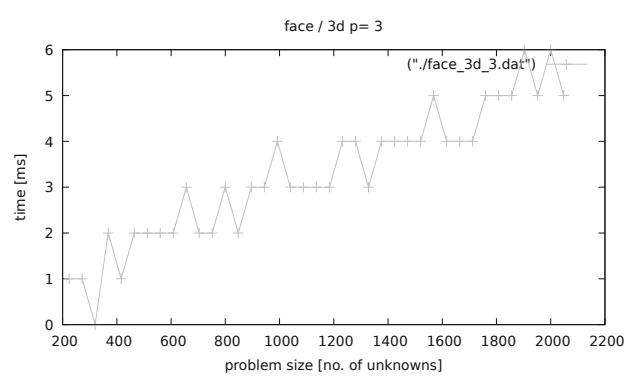

Fig. 33. Scalability of sequential hypergraph grammar-based execution over the sequence of meshes refined toward the face singularity, with uniform $p=3$.

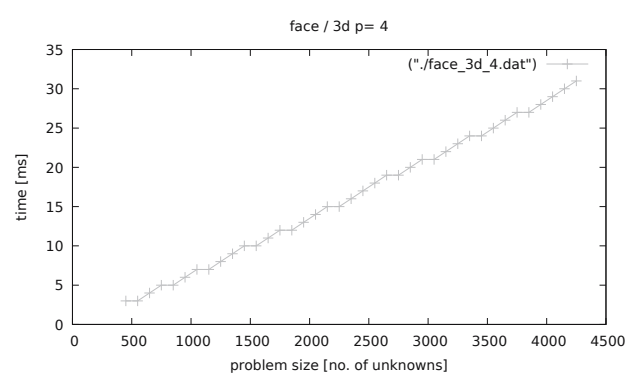

Fig. 34. Scalability of sequential hypergraph grammar-based execution over the sequence of meshes refined toward the face singularity, with uniform $p=4$.

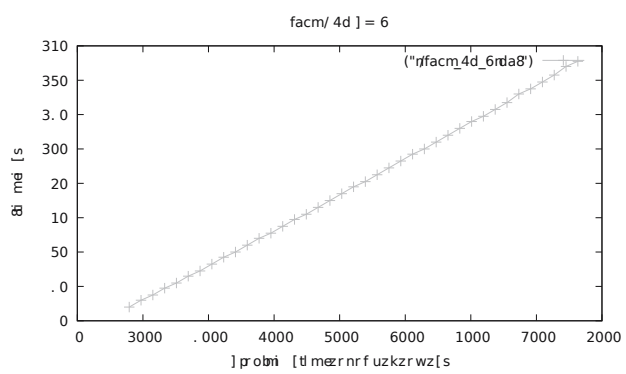

Fig. 35. Scalability of sequential hypergraph grammar-based execution over the sequence of meshes refined toward the face singularity, with uniform $p=5$. 


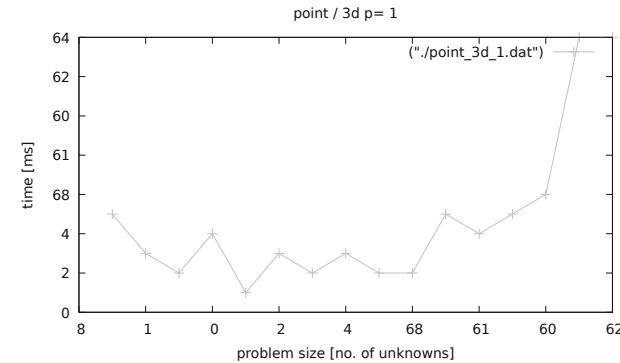

Fig. 36. Scalability of parallel hypergraph grammar-based execution over the mesh refined toward the point singularity, with uniform $p=2$.

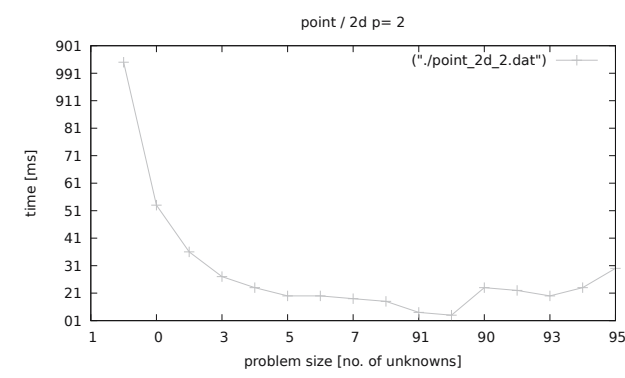

Fig. 37. Scalability of parallel hypergraph grammar-based execution over the mesh refined toward the point singularity, with uniform $p=3$.

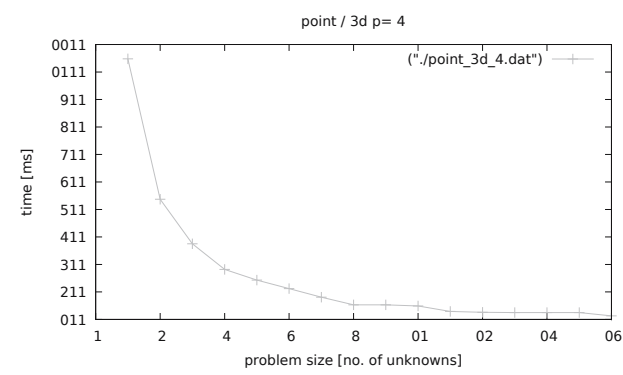

Fig. 38. Scalability of parallel hypergraph grammar-based execution over the mesh refined toward the point singularity, with uniform $p=4$.

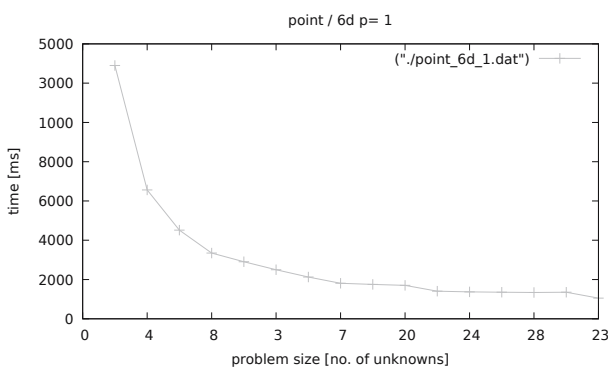

Fig. 39. Scalability of parallel hypergraph grammar-based execution over the mesh refined toward the point singularity, with uniform $p=5$.

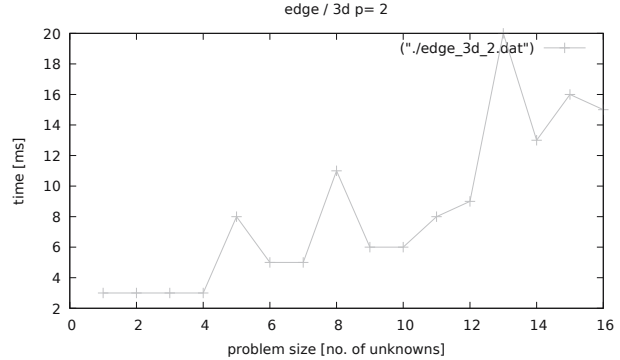

Fig. 40. Scalability of parallel hypergraph grammar-based execution over the mesh refined toward the edge singularity, with uniform $p=2$.

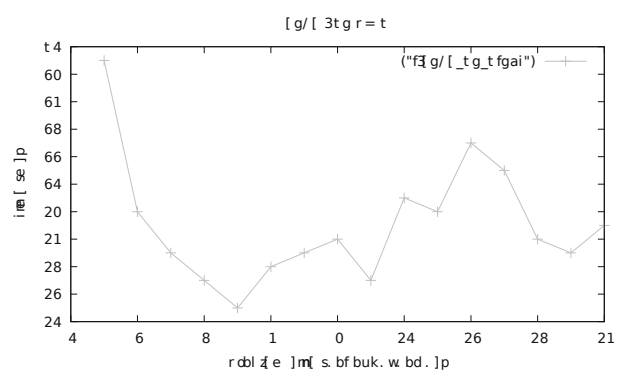

Fig. 41. Scalability of parallel hypergraph grammar-based execution over the mesh refined toward the edge singularity, with uniform $p=3$.

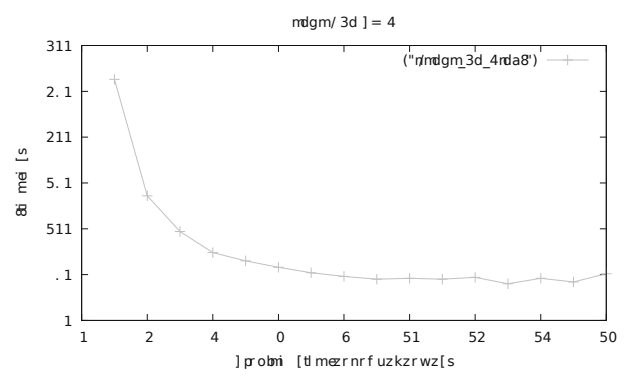

Fig. 42. Scalability of parallel hypergraph grammar-based execution over the mesh refined toward the edge singularity, with uniform $p=4$.

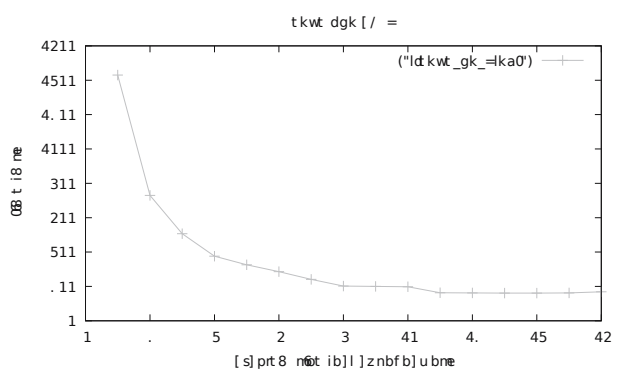

Fig. 43. Scalability of parallel hypergraph grammar-based execution over the mesh refined toward the edge singularity, with uniform $p=5$. 


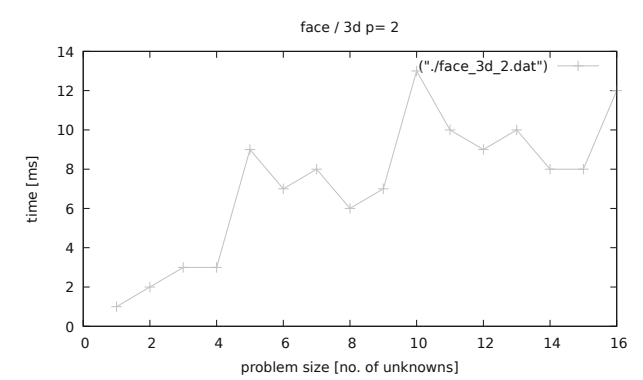

Fig. 44. Scalability of parallel hypergraph grammar-based execution over the mesh refined toward the face singularity, with uniform $p=2$.

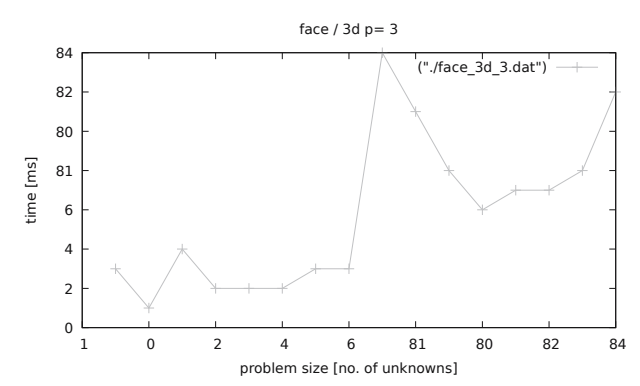

Fig. 45. Scalability of parallel hypergraph grammar-based execution over the mesh refined toward the face singularity, with uniform $p=3$.

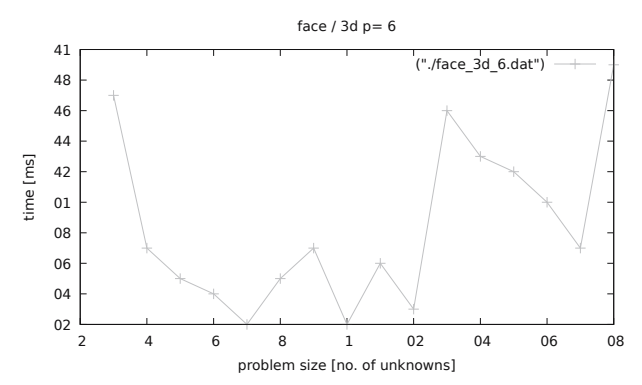

Fig. 46. Scalability of parallel hypergraph grammar-based execution over the mesh refined toward the face singularity, with uniform $p=4$.

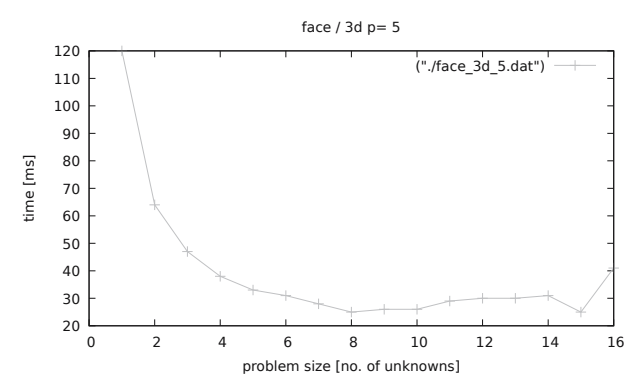

Fig. 47. Scalability of parallel hypergraph grammar-based execution over the mesh refined toward the face singularity, with uniform $p=5$. we analyzed three-dimensional meshes refined towards a point, an edge and a face. We proved theoretically and experimentally the linear computational cost of the algorithm. We also showed the theoretical logarithmic computational cost of the parallel execution, especially for polynomial orders of approximation $p>2$, and we have verified the parallel scalability on a shared-memory Linux cluster node with 16 cores. The scalability of the hypergraph-grammar-based solvers does not depend on the PDE being solved, but rather on the structure of the computational mesh generated by the $h$ adaptive procedure.

\section{Acknowledgment}

The work presented in this paper is supported by the Polish National Science Center (grant no. DEC-2015/17/B/ST6/01867).

\section{References}

Aboueisha, H., Calo, V.M., Jopek, K., Moshkov, M., Paszyńska, A., Paszyński, M. and Skotniczny, M. (2017). Element partition trees for $h$-refined meshes to optimize direct solver performance. Part I: Dynamic programming, International Journal of Applied Mathematics and Computer Science 27(2): 351-365, DOI: 10.1515/amcs-2017-0025.

Bao, G., Hu, G. and Liu, D. (2012). An $h$-adaptive finite element solver for the calculations of the electronic structures, Journal of Computational Physics 231(14): 4967-4979.

Belytschko, T. and Tabbar, M. (1993). $h$-adaptive finite element methods for dynamic problems, with emphasis on localization, International Journal for Numerical Methods in Engineering 36(24): 4245-4625.

Duff, I.S. and Reid, J.K. (1983). The multifrontal solution of indefinite sparse symmetric linear, ACM Transactions on Mathematical Software 9(3): 302-325.

Duff, I.S. and Reid, J.K. (1984). The multifrontal solution of unsymmetric sets of linear equations, SIAM Journal on Scientific and Statistical Computing 5(3): 633-641.

Flasiński, M. and Schaefer, R. (1996). Quasi context sensitive graph grammars as a formal model of FE mesh generation, Computer-Assisted Mechanics and Engineering Science 3: 191-203.

Goik, D., Paszyński, M., Lenharth, A., Nguyen, D. and Pingali, K. (2014). Graph grammar based multi-thread multi-frontal direct solver with Galois scheduler, Procedia Computer Science 29: 960-969.

Grabska, E. (1993a). Theoretical concepts of graphical modeling. Part I: Realization of $\mathrm{CP}$-graphs, Machine Graphics and Vision 1(2): 3-38.

Grabska, E. (1993b). Theoretical concepts of graphical modeling. Part II: CP-graph grammars and languages, $\mathrm{Ma}$ chine Graphics and Vision 2(2): 149-178. 
Habel, A. and Kreowski, H.J. (1987a). May we introduce to you: Hyperedge replacement, in H. Ehrig et al. (Eds.), GraphGrammars and Their Application to Computer Science, Lecture Notes in Computer Science, Vol. 291, Springer, Berlin/Heidelberg, pp. 5-26.

Habel, A. and Kreowski, H.J. (1987b). Some structural aspects of hypergraph languages generated by hyperedge replacement, in F.J. Brandenburg et al. (Eds.), STACS 87, Lecture Notes in Computer Science, Vol. 247, Springer, Berlin/Heidelberg, pp. 207-219.

Irons, B.M. (1970). A frontal solution program for finite-element analysis, International Journal for Numerical Methods in Engineering 2: 5-32.

Karypis, G. and Kumar, V. (2009). MeTis: Unstructured Graph Partitioning and Sparse Matrix Ordering System, Version 4.0, http: / / www. cs. umn. edu/ metis

Paszyńska, A., Grabska, E. and Paszyński, M. (2012a). A graph grammar model of the $h p$ adaptive three dimensional finite element method, Part I, Fundamenta Informaticae 114(2): 149-182.

Paszyńska, A., Grabska, E. and Paszyński, M. (2012b). A graph grammar model of the HP adaptive three dimensional finite element method, Part II, Fundamenta Informaticae 114(2): 183-201.

Paszyńska, A., Paszyński, M. and Grabska, E. (2009). Graph transformations for modeling $h p$-adaptive finite element method with mixed triangular and rectangular elements, in G. Allen et al. (Eds.), ICCS 2009, Lecture Notes in Computer Science, Vol. 5545, Springer, Berlin/Heidelberg, pp. 875-884.

Paszyńska, A., Paszyński, M., Jopek, K., Woźniak, M., Goik, D., Gurgul, P., AbouEisha, H., Moshkov, M., Calo, V.M., Lenharth, A., Nguyen, D. and Pingali, K. (2015). Quasi-optimal elimination trees for 2D grids with singularities, Scientific Programming 2015, Article ID: 303024, DOI:10.1155/2015/303024.

Paszyński, M. (2009). On the parallelization of self-adaptive $h p$-finite element methods, Part I: Composite programmable graph grammar model, Fundamenta Informaticae 4(93): 411-434.

Paszyński, M. (2016). Fast Solvers for Mesh-Based Computations, CRC Press, Boca Raton, FL.

Paszyński, M. and Paszyńska, A. (2008). Graph transformations for modeling parallel $h p$-adaptive finite element method, in R. Wyrzykowski et al. (Eds.), PPAM 2007, Lecture Notes in Computer Science, Vol. 4967, Springer, Berlin/Heidelberg, pp. 1313-1322.

Paszyński, M. and Schaefer, R. (2010). Graph grammar-driven parallel partial differential equation solver, Concurrency and Computation Practice and Experience 22: 1063-1097.

Pingali, K., Nguyen, D., Kulkarni, K., Burtscher, K.M., Hassaan, M.A., Kaleem, R., Lee, T.-H., Lenharth, A., Manevich, R., Mendez-Lojo, M., Prountzos, D. and Sui, X. (2011). The Tao of parallelism in algorithms, 32nd ACM SIGPLAN Conference on Programming Language Design and Implementation, San Jose, CA, USA, pp. 12-22.
Ryszka, I., Paszyńska, A., Grabska, E., Sieniek, M. and Paszyński, M. (2015a). Graph transformation systems for modeling three dimensional finite element method, Part I, Fundamenta Informaticae 140(2): 129-172.

Ryszka, I., Paszyńska, A., Grabska, E., Sieniek, M. and Paszyński, M. (2015b). Graph transformation systems for modeling three dimensional finite element method, Part II, Fundamenta Informaticae 140(2): 173-203.

Ślusarczyk, G. and Paszyńska, A. (2013). Hypergraph grammars in $h p$-adaptive finite element method, Procedia Computer Science 18: 1545-1554.

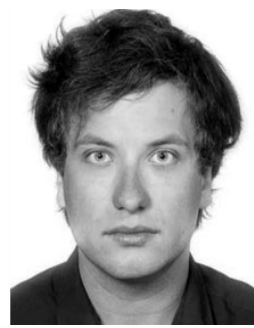

Piotr Gurgul was born in 1987. He graduated from a combined MSc and BEng program in computer science at the AGH University of Science and Technology in Kraków in 2011, and from a BBA program in management from the Kraków University of Economics in 2012. In 2014 he obtained his $\mathrm{PhD}$ in computer science at the AGH University of Science and Technology. His scientific interests focus on the hp-adaptive finite element method and adaptive solvers. Currently he works at Dropbox Inc. in California.

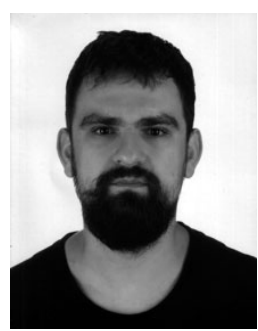

Konrad Jopek is a $\mathrm{PhD}$ student at the Department of Computer Science of the AGH University of Science and Technology. He is a co-author of several publications about fast solvers for the finite element method. His research interests focus on numerical methods, parallel algorithms, highly-scalable software and low-level progamming. His $\mathrm{PhD}$ advisors are Maciej Paszyński and Anna Paszyńska.

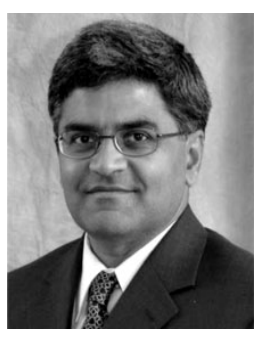

Keshav Pingali is a W.A."Tex" Moncrief Chair of Grid and Distributed Computing, a professor at the Department of Computer Science and the Institute for Computational Engineering and Sciences, University of Texas, Austin. His current research interests include methodologies and tools for programming multicore processors, with a focus on irregular applications from domains like graphics, social networks, and data mining.

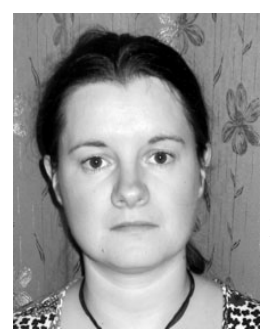

Anna Paszyńska received her $\mathrm{PhD}$ (2007) in computer science from the Institute of Fundamental Technological Research of the Polish Academy of Sciences in Warsaw, Poland. She currently works as an assistant professor at the Faculty of Physics, Astronomy and Applied Computer Science, Jagiellonian University in Kraków, Poland. Her research interests include evolutionary algorithms, graph grammar and computer aided design.

Received: 13 June 2017

Revised: 24 January 2018

Re-revised: 10 March 2018

Accepted: 19 April 2018 